\title{
La colaboración científica en el campo de las Ciencias de la Actividad Física y el Deporte en España
}

\author{
Javier Valenciano Valcárcel ${ }^{\star}$, José Devís-Devís**, \\ Miguel Villamón ${ }^{\star \star}$, Carmen Peiró-Velert ${ }^{\star \star \star}$
}

Resumen: Este estudio examina la colaboración científica a través de los patrones de coautoría en una muestra de revistas españolas de Ciencias de la Actividad Física y el Deporte (CCAFD). También analiza los principales grupos de investigación y disciplinas del campo multidisciplinar de las CCAFD. Se analizaron la frecuencia y porcentaje de los artículos en colaboración y el número de autores por artículo. Se revisaron las redes de colaboración de los autores más productivos para identificar grupos de investigación y se calculó la densidad para conocer su cohesión. La producción científica fue analizada también por disciplinas. Los artículos en colaboración superan a los artículos de autor único en la mayoría de revistas y disciplinas del campo español de las CCAFD. El promedio de autores por artículo es de 2,50. Se identificaron 15 grupos de investigación y los más numerosos son los más productivos y los menos cohesionados. La colaboración con autores extranjeros en las revistas españolas de la muestra es baja y la mayoría se limita a autores suramericanos. La Didáctica y la Teoría del Entrenamiento son las disciplinas más productivas. Los resultados muestran la tendencia creciente hacia la colaboración observada durante los últimos años en las CCAFD. No obstante, la colaboración es todavía baja en cuanto al número de autores por artículo y al número de grupos.

Palabras clave: Colaboración científica, grupos de investigación, coautoría, Ciencias de la Actividad Física y el Deporte, España.

\section{Scientific cooperation in the field of Physical Activity and Sport Science in Spain}

Abstract: This study examines scientific cooperation through co-authorship patterns in a sample of Spanish academic Physical Activity and Sport Science (PASS) journals. It also examines the main research groups and disciplines of the multidisciplinary PASS field. The frequency and percentage of jointly authored articles, and the number of authors per paper, were analysed. Cooperative network analysis was performed for the most productive authors to identify research groups, and the group density was calculated to reveal group cohesion. Production was also analysed by discipline. Single-authored

* Facultad de Educación. Toledo. Universidad de Castilla-La Mancha. Correo-e: Javier.Valenciano@uclm.es.

*** Facultad de Ciencias de la Actividad Física y el Deporte. Universitat de València. Correo-e: Jose.Devis@uv.es, Miguel.Villamon@uv.es.

*** E. U. de Magisterio. Universitat de València. Correo-e: Carmen.Peiro@uv.es.

Recibido: 25-5-09; 2. ${ }^{a}$ versión: 15-7-09; aceptado: 3-8-09. 
publications were fewer than co-authored publications in most of the journals and disciplines examined. The average number of authors per article was 2.5. Fifteen research groups were identified, the larger ones being the most productive and less cohesive. Cooperation with foreign researchers was modest and mostly limited to South American authors. Teaching and training theory were the most productive disciplines. The results highlight the trend towards cooperation that has been observed over recent years in PASS, but cooperation in terms of the number of co-authors per article remains rather low, as does the number of research groups identified.

Keywords: Scientific cooperation, research groups, co-authorship, Physical Activity and Sport Science, Spain.

\section{Introducción}

La institucionalización de la ciencia moderna durante el siglo XVII en Europa supuso el reconocimiento formal de la comunidad científica y propició el establecimiento de unas incipientes relaciones de colaboración entre los investigadores. Desde entonces, la colaboración entre científicos de distintas instituciones, países o campos de especialización es una práctica habitual para avanzar en la construcción y reconstrucción del conocimiento. El aumento de la colaboración científica ha sido especialmente notorio en los campos de la medicina y de la ciencia y la tecnología, en particular a partir de la segunda mitad del siglo xx (Bordons, 2000; Cronin, 2001). En las ciencias sociales se ha observado asimismo un aumento creciente de la colaboración, si bien es cierto que algunas disciplinas humanísticas aún preservan una cultura científica marcadamente individualista (Cronin, 2003). La colaboración es una práctica extendida y, en muchos casos, una necesidad derivada de la complejidad de los desafíos que el propio avance del conocimiento plantea. En este sentido, las políticas científicas de los distintos países incentivan la cooperación para optimizar tanto los recursos y las inversiones como los resultados de la investigación.

La colaboración científica, como práctica social, ha sido analizada en múltiples contextos geográficos y disciplinares (Alonso-Arroyo, 2005; Arunachalam, 2000; Glänzel, 1999; Olmeda-Gómez, 2008; Wang, 2005). Este aspecto de la actividad científica tiene especial interés en el contexto español de las Ciencias de la Actividad Física y el Deporte (CCAFD), puesto que la colaboración puede redundar en un aumento de la producción científica y, en el caso de las colaboraciones con investigadores de otros países, en una oportunidad para la proyección internacional de los investigadores españoles. La proyección de la comunidad científica española debería incluir la internacionalización de las revistas, de manera que al tiempo que se incrementara la visibilidad de los autores en revistas internacionales, los títulos españoles también reflejaran los resultados de esas relaciones de colaboración.

En el ámbito internacional de las CCAFD se han realizado trabajos sobre distintos aspectos de la producción científica como el contenido, los temas o áreas de estudio y las tendencias de las publicaciones (Heinemann, 1990; Lidor, 1999; 
Thompson, 1996; Ward, 2006; Yaman, 2007). En España se han estudiado las características y la calidad de las revistas españolas de este campo de conocimiento en expansión con el objeto de contribuir a la mejora de estos instrumentos de comunicación científica (Valenciano, 2008a, 2008b; Villamón, 2007). Se trataba de una necesidad ligada al aumento de la actividad científica que se ha producido como consecuencia de la incorporación progresiva de la comunidad científica de las CCAFD al sistema universitario español durante las dos últimas décadas. De hecho, la Universidad española soporta el grueso de la investigación en este campo, como refleja el hecho de que el 87,29\% de la producción científica publicada en sus revistas esté rubricada por esta institución (Valenciano, 2009). En cambio, hay pocas aportaciones que hayan analizado la colaboración científica (Calatayud, 2000). Además, es necesario abarcar una muestra de publicaciones más amplia porque la mayoría de los trabajos han utilizado una muestra insuficiente para analizar los patrones de la colaboración científica en un campo multidisciplinar como el de las CCAFD. Para ello se ha planteado este trabajo, que examina la colaboración científica en las CCAFD a través de la coautoría reflejada en una muestra de revistas españolas del campo.

\section{Metodología}

Las publicaciones sobre las que se analizó la colaboración fueron seleccionadas a partir de una evaluación de las revistas españolas de CCAFD publicada recientemente (Valenciano, 2008b). Como primera aproximación a la colaboración científica en este campo de conocimiento y, dada la escasa contribución de los autores españoles en revistas internacionales detectada en fases preliminares del proyecto al que pertenece este estudio (ver Agradecimientos), se consideró suficiente delimitar la muestra sobre revistas españolas. En concreto, de los 28 títulos evaluados en el trabajo referido, la muestra quedó integrada por las 16 revistas que obtuvieron los mejores resultados. De esta manera, el estudio se realiza sobre las revistas mejor consideradas por los investigadores para la difusión de sus trabajos. El período de análisis establecido fue 1999-2005, ambos años incluidos.

La recogida de los datos se realizó a través de la consulta directa de los ejemplares de las revistas y solo se consideraron los trabajos originales. En una base de datos, confeccionada ad hoc en Microsoft Acces 2000, se registraron los siguientes campos: fuente (revista, número y año), título del artículo y disciplina, autores y países. La disciplina fue asignada mediante un proceso de categorización inductiva a partir del título del trabajo, del resumen y de las palabras clave. Entre las distintas alternativas para el conteo, se optó por un recuento total o completo de los autores porque otorga el mismo reconocimiento a todos los firmantes, con independencia del número y del orden de las firmas, y no penaliza la colaboración.

En la base de datos, se programaron una serie de consultas cuyos informes se exportaron a una hoja de cálculo Microsoft Excel 2000 para completar el análisis. Como indicadores de la colaboración se analizaron la frecuencia de artícu- 
los firmados en coautoría y el número de autores por trabajo. Estos análisis se efectuaron para el conjunto de la producción científica, para cada revista y disciplina y para los autores más productivos. También se identificaron grupos de investigación a partir de la metodología establecida y validada por Bordons y colaboradores (Bordons, 1995; Zulueta, 1999). La delimitación de los grupos se realizó a través de una aplicación informática que ordena los autores según su producción científica. A partir del autor más productivo, se rastreó la frecuencia de las colaboraciones establecidas con otros autores y se identificaron los miembros de los diferentes grupos conforme a los siguientes criterios: 1) El autor más productivo es considerado como el investigador principal y debe haber publicado anualmente un artículo como mínimo; 2) Los autores ocasionales, aquellos que sólo han publicado un artículo en el período analizado, no se tienen en cuenta; 3) Para ser incluido en un grupo, un autor debe firmar, al menos, el 60\% de su producción con el autor principal; 4) Un grupo debe estar integrado por un mínimo de tres investigadores.

Alguno de estos criterios fueron adaptados para su aplicación al campo de las CCAFD en España, tal y como se explica a continuación. No fue necesario establecer un mínimo de publicaciones para el autor principal puesto que el análisis se realizó sobre los autores más productivos, esto es, aquellos que han publicado un mínimo de 10 artículos. Asimismo, la producción mínima que cualquier autor debe firmar junto al investigador principal se redujo del $60 \%$ al $50 \%$, al detectar los bajos niveles de colaboración en el contexto analizado y que esta reducción no provocaría una fragmentación de los grupos.

Para conocer la cohesión de los grupos se calculó la densidad. Este indicador se usa en el análisis de redes sociales para conocer el grado de conexión de la red, según la relación entre el número de enlaces establecidos y el número de enlaces posibles entre los miembros del grupo (Otte, 2002; Valderrama-Zurián, 2007). Se expresa así:

$$
\text { Densidad }=\frac{2 e}{n(n-1)}
$$

Donde:

$e=\mathrm{n}^{\circ}$ de enlaces entre los miembros del grupo, $\mathrm{y}$

$n=\mathrm{n}^{\circ}$ de miembros.

\section{Resultados y discusión}

\subsection{Coautoría y número de autores por trabajo en el conjunto de la producción}

En el período comprendido entre 1999 y 2005 se publicaron un total de 1.786 artículos en las 16 revistas de la muestra. La mayoría de ellos, el 63,94\% (1.142), se firmaron por varios autores. El número de autores diferentes asciende a 2.251 
y el total de firmas plasmadas a 4.462, lo que supone una media de autores por trabajo de 2,50.

Estos resultados muestran que la colaboración es una práctica común en las CCAFD en España durante el período analizado. Aunque el porcentaje de artículos firmados en coautoría no alcanza porcentajes característicos de las Ciencias de la Salud, como el 86,34\% de la Dermatología (Aleixandre, 2003) o el 95,1\% de la Cardiología (Valderrama, 2007), se sitúa por encima del 32,8\% observado en el ámbito la Educación (Vallejo, 2005), del 19,47\% (López, 2001) de la Documentación o del 13,96\% de la Sociología (López, 1999). Del mismo modo, el número de autores por trabajo está por debajo de los 4 de las Ciencias de la Salud, la Ingeniería y la Química (Bordons, 1997), que alcanza un valor en torno a 6 en disciplinas médicas como en el área cardiovascular (Valderrama, 2007; Zulueta, 1999) e incluso de 9 en el ámbito de la Física (Bordons, 1997). En cambio, es algo superior al de las Humanidades y las Ciencias Sociales, que se sitúa alrededor de 1 y de 2 (Bordons, 1997; Cronin, 2003), y supera en más de un punto al 1,3 de la Documentación (Jiménez, 1997), al 1,18 de la Sociología y al 1,14 de la Ciencia Política (López, 1999).

El análisis diacrónico de la coautoría revela una fluctuación de los trabajos realizados en colaboración a lo largo del período analizado. En la figura 1 se observa un descenso desde el inicio del período, cuando el 78,24\% de las contribuciones fueron rubricadas por más de un autor. El punto de inflexión de esta tendencia se produce a mitad del período, en 2002, cuando el porcentaje de trabajos en colaboración fue del 59,94\%. A partir de ese momento, se aprecia un tímido aumento que alcanza el $71,25 \%$ en el último año.

\section{FIGURA 1}

Porcentaje de articulos en colaboración entre 1999 y 2005

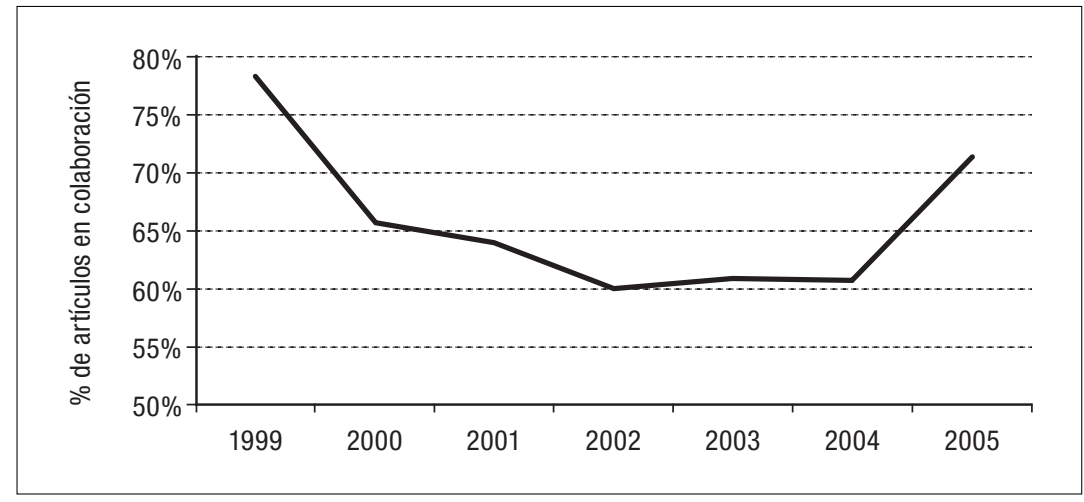

Por el contrario, el número de autores por trabajo se mantiene más estable a lo largo de los 7 años, tal y como se refleja en la figura 2. Se observa un valor 
mínimo de 2,34 en el año 2002 y un valor máximo de 2,74 en 2005, al final de período.

FIGURA 2

Promedio de autores por artículo entre 1999 y 2005

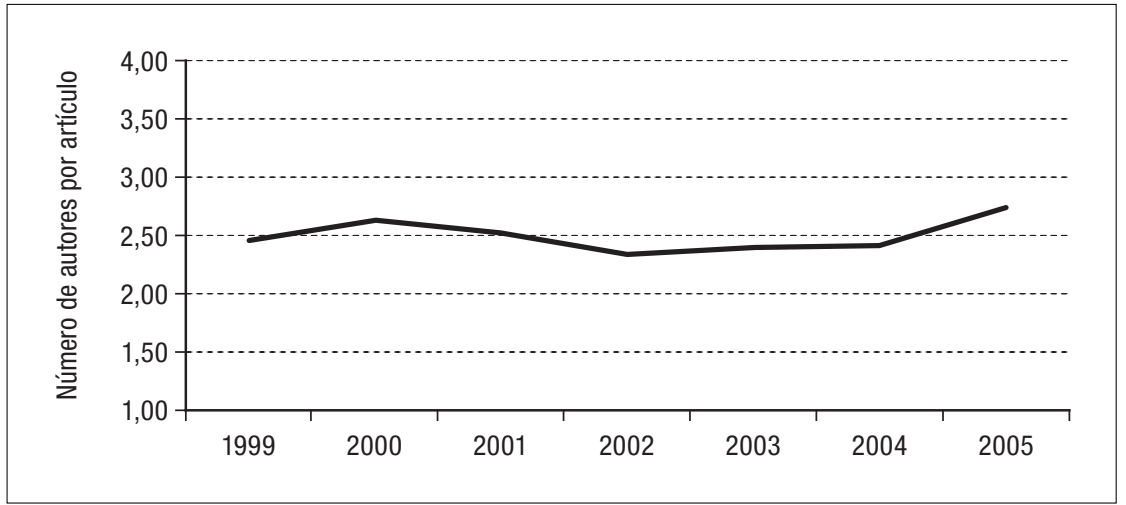

La figura 3 muestra cómo la cantidad de trabajos disminuye a medida que aumenta el número de coautores. Aunque la frecuencia más elevada corresponde a los artículos firmados por un único autor (644, el 36,06\%), no supera el total de trabajos firmados en colaboración (1142, el 63,94\%). El 24,36\% de los trabajos están firmados por dos autores, el 15,62\% por tres, el 11,53\% por cuatro y el 6,83\% por cinco. Los artículos firmados por cinco o más autores sólo suponen el 5,60\% del total.

FIGURA 3

Frecuencia de artículos por número de coautores

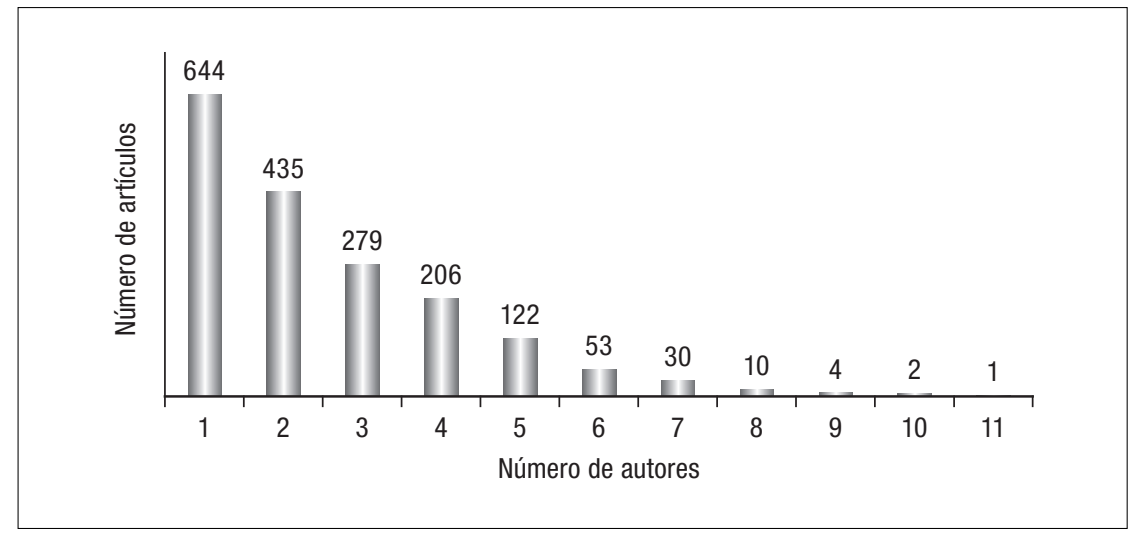


Hay que destacar que, de los 2251 autores, solamente 36 han publicado 10 o más trabajos. El análisis de la producción científica de estos autores arroja un porcentaje de trabajos realizados en colaboración y un número de autores por trabajo superiores a los observados en el conjunto de la producción. De hecho, el 94,02\% de los trabajos de estos autores están firmados en colaboración. Además, el $75 \%$ de ellos ha realizado el 100\% de sus trabajos con otros autores, es decir, no ha firmado individualmente ni una sola contribución. De la misma manera, el número de autores por trabajo para la producción científica de esos autores es de 3,89. La mitad de ellos posee una producción científica que arroja un número de autores por trabajo superior a 4 y en tres casos alcanza o sobrepasa los 5. Solamente dos firman artículos con un número de autores por trabajo inferior a la media $(2,50)$. Estos datos sugieren que la cooperación entre los investigadores redunda en una mayor productividad además de que, como señala la literatura, confiera crédito científico a los trabajos y contribuya a su repercusión a través de la citación (Borgman, 2002; Glänzel, 2002).

Respecto a la participación de investigadores de otros países, se han contabilizado 369 autores extranjeros, lo que supone el 16,39\% del total. La mayoría de ellos proceden de Brasil (67), Portugal (51) y Argentina (40). Entre los autores extranjeros, los latinoamericanos ascienden a 231 (el 62,60\%), mientras que los europeos suman 102 (el 27,64\%). Los principales países europeos de procedencia son, además de Portugal, Reino Unido (11), Italia (10), Bélgica (9) y Francia (9). También han contribuido autores de otros países de habla inglesa como Estados Unidos (13), Canadá (9) y Australia (6). Aunque la participación de autores extranjeros ha tenido lugar en el 12,77\% de los trabajos, la colaboración entre éstos y los autores españoles se ha producido solamente en el 3,25\%, ya que el resto de las aportaciones han sido firmadas exclusivamente por los primeros. Estos resultados reflejan que las colaboraciones establecidas entre investigadores españoles y extranjeros son muy escasas y que la mayoría de ellas se producen con investigadores de países cultural o geográficamente próximos, como los latinoamericanos o Portugal. En cambio, y a pesar de que algunas revistas aceptan manuscritos en inglés, las colaboraciones establecidas con investigadores de países de habla inglesa son muy discretas. En todo caso, conviene advertir que el hecho de que la muestra analizada se limite a revistas españolas puede influir en los datos anteriores. De hecho, es probable que los resultados de esas colaboraciones tiendan a publicarse preferiblemente en revistas europeas o americanas antes que en títulos españoles para alcanzar una visibilidad y reconocimiento mayores, puesto que las revistas españolas tienen una proyección internacional modesta. Aunque el $44,5 \%$ de las revistas incluidas en el Catálogo Latindex están indizadas por alguna base de datos internacional, solamente dos figuran en las bases de Thomson-Reuters y una en Scopus (Villamón, 2009).

El examen de la producción de cada una de las revistas denota que publican mayoritariamente artículos en colaboración. Como se observa en la Tabla I, solamente tres revistas tienen un porcentaje de artículos en colaboración por debajo del 50\%: Revista Española de Educación Física y Deportes, Revista Iberoamerica- 
na de Psicomotricidad y Técnicas Corporales y Tándem. Las dos últimas revistas son, asimismo, las únicas cuyo número de autores por trabajo es inferior a 2.

TABLA I

Número de artículos, porcentaje de artículos en colaboración y promedio de autores por artículo en cada revista

\begin{tabular}{|c|c|c|c|}
\hline Revista & $\begin{array}{l}\text { Número } \\
\text { de artículos }\end{array}$ & $\begin{array}{c}\% \\
\text { artículos en } \\
\text { colaboración }\end{array}$ & $\begin{array}{c}\text { Autores / } \\
\text { artículo }\end{array}$ \\
\hline $\begin{array}{l}\text { Motricidad. European Journal of Human Mo- } \\
\text { vement }\end{array}$ & 87 & 85,06 & 3,38 \\
\hline Archivos de Medicina del Deporte & 273 & 82,78 & 3,47 \\
\hline $\begin{array}{l}\text { Selección. Revista Española e Iberoamericana } \\
\text { de Medicina de la Educación Física y el } \\
\text { Deporte }\end{array}$ & 124 & 80,65 & 2,95 \\
\hline Revista de Entrenamiento Deportivo (RED) & 126 & 76,98 & 2,83 \\
\hline Apunts. Medicina del Deporte & 87 & 75,86 & 3,32 \\
\hline Revista de Psicología del Deporte & 113 & 74,34 & 2,42 \\
\hline $\begin{array}{l}\text { Kronos. La revista cientifica de actividad fisi- } \\
\quad \text { ca y el deporte }\end{array}$ & 60 & 70,00 & 2,27 \\
\hline Cuadernos de Psicología del Deporte & 65 & 67,69 & 2,29 \\
\hline Cultura, Ciencia y Deporte & 19 & 63,16 & 2,42 \\
\hline Revista de Educación Física (REF) & 114 & 61,40 & 2,28 \\
\hline $\begin{array}{l}\text { Revista Internacional de Ciencias del Deporte } \\
\quad(\text { RICYDE) }\end{array}$ & 5 & 60,00 & 2,00 \\
\hline Apunts. Educación Física y Deporte & 302 & 55,63 & 2,08 \\
\hline $\begin{array}{l}\text { Revista Internacional de Medicina y Ciencias } \\
\quad \text { de la Actividad Física y el Deporte }\end{array}$ & 98 & 55,10 & 2,22 \\
\hline $\begin{array}{l}\text { Revista Española de Educación Física y De- } \\
\quad \text { portes }\end{array}$ & 17 & 41,18 & 2,00 \\
\hline $\begin{array}{l}\text { Revista Iberoamericana de Psicomotricidad y } \\
\text { Técnicas Corporales }\end{array}$ & 112 & 32,14 & 1,59 \\
\hline Tándem. Didáctica de la Educación Física & 184 & 32,07 & 1,51 \\
\hline
\end{tabular}

\subsection{Coautoría y número de autores por trabajo en cada disciplina}

Los artículos de la muestra de revistas analizada pertenecen a 22 disciplinas diferentes, lo que pone de manifiesto el carácter multidisciplinar del campo de las CCAFD. No obstante, la mitad de las disciplinas no alcanzan los 50 artículos 
y en cuatro de ellas las contribuciones han sido ocasionales (Ética, Ingeniería, Ciencias de la Información y Filología). Las disciplinas más productivas, con un número de trabajos por encima de 100, son la Didáctica (381 artículos; 21,33\% del total), la Teoría del Entrenamiento (324 artículos; 18,14\%), la Psicología (242 artículos; 13,55\%), la Medicina (216 artículos, 12,09\%) y la Fisiología (130 artículos, 7,28\%). La Didáctica y la Teoría del Entrenamiento aparecen como los principales focos de investigación debido a que tanto la enseñanza como el entrenamiento deportivo son los ámbitos profesionales más característicos de las CCAFD. La Psicología ha sido otra disciplina frecuente entre las CCAFD, que ha aplicado sus teorías y métodos para optimizar los procesos de enseñanza-aprendizaje de las actividades físico-deportivas y el rendimiento deportivo. Del mismo modo, las Ciencias de la Salud han desempeñado un papel prominente en la expansión del conocimiento científico en este campo, además de que son disciplinas tradicionalmente prolíficas. De hecho, las diferentes disciplinas de las Ciencias de la Salud reúnen entre sí el 24,13\% de la producción científica analizada.

Por lo que se refiere a la colaboración, la coautoría está por debajo del 50\% en el caso de las disciplinas de las Humanidades y las Ciencias Sociales como la Didáctica (42,50\%), la Psicomotricidad (32,86\%), la Teoría de la Educación Física y el Deporte (20\%), la Historia (17,14\%) y la Antropología (6,67\%) (ver Tabla II). Estos resultados oscilan entre unos márgenes similares a los obtenidos en otras disciplinas de las Humanidades y las Ciencias Sociales como la Educación (30\%) o la Filosofía (5\%) (Cronin, 2003; Vallejo, 2005). La Psicología ha alcanzado el $71,9 \%$ de coautoría y representa la tendencia hacia mayores niveles de colaboración, tal cual ha observado Cronin (2003) en la ciencia en general. Las disciplinas con más del 75\% de los trabajos firmados en coautoría son la Cinesiología (96\%), la Fisiología (91,54\%), la Antropometría (86,36\%), la Actividad Física y Salud (82,93\%), la Biomecánica (81,43\%), la Teoría del Entrenamiento (76,54\%) y la Medicina (75,46\%). Estos porcentajes están próximos a los observados en otras ocasiones en el ámbito de la Medicina, la Biología o la Química, por ejemplo (Cronin, 2004; Newman, 2004, Valderrama-Zurián, 2007).

Respecto al número de autores por trabajo, las disciplinas de las Humanidades y las Ciencias Sociales identificadas entre las CCAFD muestran patrones que les son propios, con un número de autores que oscila entre 1 y 2 (Cronin, 2003). En concreto, en la Antropología se observa un número de autores por trabajo de 1,07, en la Historia de 1,20, en la Teoría de la Educación Física y el Deporte de 1,26, en la Psicomotricidad de 1,50, en la Sociología de 1,78, en la Didáctica de 1,81 y en la Gestión de 1,83. Las disciplinas de las Ciencias de la Salud ofrecen, en cambio, los valores más altos. La mayoría de estas disciplinas tiene un número de autores por trabajo de entre 3 y 4 como, por ejemplo, la Medicina (3), la Antropometría (3,39), la Cinesiología (3,64) y la Fisiología $(3,87)$. A pesar de ello, estos valores están lejos de los observados en otros trabajos del campo de la Medicina, donde el promedio de autores por trabajo es de 5 o 6 autores (Drenth, 1998; Valderrama-Zurián, 2007). 


\section{TABLA II}

Número de artículos, porcentaje de artículos en colaboración y promedio de autores por artículo en cada disciplina

\begin{tabular}{l|c|c|c}
\hline \multicolumn{1}{c|}{ Disciplina } & $\begin{array}{c}\text { Número } \\
\text { de artículos }\end{array}$ & $\begin{array}{c}\text { \% } \\
\text { artículos en } \\
\text { colaboración }\end{array}$ & $\begin{array}{c}\text { Autores / } \\
\text { artículo }\end{array}$ \\
\hline Cinesiología & 25 & 96,00 & 3,64 \\
\hline Fisiología & 130 & 91,54 & 3,87 \\
\hline Antropometría & 44 & 86,36 & 3,39 \\
\hline Actividad física y salud & 41 & 82,93 & 2,83 \\
\hline Biomecánica & 70 & 81,43 & 3,33 \\
\hline Teoría del Entrenamiento & 324 & 76,54 & 2,85 \\
\hline Medicina & 216 & 75,46 & 3,00 \\
\hline Psicología & 242 & 71,90 & 2,38 \\
\hline Documentación & 19 & 63,16 & 2,47 \\
\hline Gestión & 41 & 58,54 & 1,83 \\
\hline Educación Social & 14 & 57,14 & 2,29 \\
\hline Sociología & 32 & 50,00 & 1,78 \\
\hline Nutrición & 16 & 50,00 & 2,56 \\
\hline Didáctica & 381 & 42,52 & 1,81 \\
\hline Psicomotricidad & 70 & 32,86 & 1,50 \\
\hline Teoría de la Educación Física y el Deporte & 35 & 20,00 & 1,26 \\
\hline Historia & 35 & 17,14 & 1,20 \\
\hline Antropología & 15 & 6,67 & 1,07 \\
\hline
\end{tabular}

\subsection{Grupos de investigación}

El análisis de las redes de colaboración de los autores más productivos ha identificado 15 grupos de investigación cuyas características principales se muestran en la Tabla III. El número medio de miembros por grupo es de 6,13 pero el tamaño más frecuente es el de tres. El tamaño de los grupos solamente ha superado la decena en dos de ellos, que han reunido 13 investigadores cada uno. El resto de grupos oscila entre los tres investigadores, el mínimo exigido, y los ocho. Cabe destacar que entre los 92 autores que integran la totalidad de los grupos no hay ni un solo autor extranjero. El tamaño medio de estos grupos es inferior al 10,92 constatado a través de una metodología similar en el campo de la Medicina (Zulueta, 1999). En ese caso, el número de miembros de algunos grupos alcanza la veintena y la agrupación más frecuente es la que reúne 7 autores. 
TABLA III

Principales características de los grupos de investigación

\begin{tabular}{|c|c|c|c|c|c|c|}
\hline $\begin{array}{l}\text { Investigador } \\
\text { principal }\end{array}$ & $\begin{array}{l}\text { Produc- } \\
\text { ción } \\
\text { con el } \\
\text { grupo }\end{array}$ & $\begin{array}{c}\mathrm{N}^{\mathbf{o}} \\
\text { de } \\
\text { autores }\end{array}$ & $\begin{array}{l}\mathrm{N}^{\circ} \\
\text { de } \\
\text { artícu- } \\
\text { los }\end{array}$ & $\begin{array}{c}\text { Autores } \\
\text { / } \\
\text { artículo }\end{array}$ & $\begin{array}{c}\text { Densi- } \\
\text { dad }\end{array}$ & $\begin{array}{l}\text { Disciplina } \\
\text { principal }\end{array}$ \\
\hline Villa Vicente, J. G. & $88,89 \%$ & 8 & 24 & 3,25 & 0,61 & $\begin{array}{l}\text { Medicina } \\
\text { Fisiología }\end{array}$ \\
\hline López Calbet, J. A. & $100,00 \%$ & 13 & 24 & 3,42 & 0,55 & $\begin{array}{l}\text { Teoría del Entrena- } \\
\text { miento }\end{array}$ \\
\hline Aguado Jódar, X. & $90,48 \%$ & 7 & 19 & 3,63 & 0,57 & Biomecánica \\
\hline Del Valle Soto, M. E. & $94,12 \%$ & 13 & 16 & 3,81 & 0,46 & Fisiología \\
\hline Del Villar Álvarez, F. & $100 \%$ & 7 & 15 & 3,73 & 0,95 & $\begin{array}{l}\text { Teoría del Entrena- } \\
\text { miento }\end{array}$ \\
\hline $\begin{array}{l}\text { Manonelles Marque- } \\
\text { ta, P. }\end{array}$ & $100 \%$ & 6 & 15 & 3,27 & 0,60 & $\begin{array}{l}\text { Medicina } \\
\text { Fisiología } \\
\text { Teoría del Entrena- } \\
\quad \text { miento } \\
\end{array}$ \\
\hline Carrasco Páez, L. & $100 \%$ & 6 & 15 & 2,53 & 0,73 & $\begin{array}{l}\text { Fisiología } \\
\text { Teoría del Entrena- } \\
\quad \text { miento }\end{array}$ \\
\hline $\begin{array}{l}\text { Moreno Hernández, } \\
\text { F. J. }\end{array}$ & $86,67 \%$ & 5 & 13 & 2,85 & 1,00 & $\begin{array}{l}\text { Teoría del Entrena- } \\
\text { miento }\end{array}$ \\
\hline García Montes, M. E. & $73,33 \%$ & 3 & 11 & 2,09 & 1,00 & Sociología \\
\hline Márquez Rosa, S. & $55,00 \%$ & 6 & 11 & 3 & 0,73 & Psicología \\
\hline De Paz Fernández, J. A. & $60,00 \%$ & 3 & 9 & 2,11 & 1,00 & $\begin{array}{l}\text { Teoría del Entrena- } \\
\text { miento } \\
\text { Fisiología }\end{array}$ \\
\hline Calderón Montero, F. J. & $80,00 \%$ & 3 & 8 & 2,63 & 1,00 & Fisiología \\
\hline Ureña Espá, A. & $61,54 \%$ & 3 & 8 & 2,25 & 1,00 & $\begin{array}{l}\text { Teoría del Entrena- } \\
\text { miento }\end{array}$ \\
\hline Gutiérrez Sainz, A. & $72,73 \%$ & 5 & 8 & 3,50 & 1,00 & $\begin{array}{l}\text { Fisiología } \\
\text { Teoría del Entrena- } \\
\quad \text { miento } \\
\end{array}$ \\
\hline Navarro Valdivieso, F. & $63,64 \%$ & 4 & 7 & 3,43 & 1,00 & Fisiología \\
\hline
\end{tabular}

Se observa que la mayoría de los investigadores principales tiene más del $70 \%$ de su producción científica integrada en la de su grupo e, incluso, cuatro de ellos, alcanzan el 100\%. Sólo en cuatro casos la producción del autor principal en colaboración con el grupo es inferior al 70\%, aunque siempre por encima del $50 \%$. Estos resultados indican que los investigadores principales mantienen 
una producción científica baja, tanto individual como en colaboración con autores diferentes a los del grupo.

Por otra parte, se ha observado una correlación positiva entre el número de miembros del grupo y el número de trabajos publicados $(r=0,77 ; p<0,05)$. En la figura 4 puede apreciarse que los grupos más numerosos tienden a publicar un número mayor de artículos. En concreto, los grupos que han firmado, al menos, 15 trabajos están constituidos por 6 o más autores. En cambio, aquellos que no han alcanzado las 10 contribuciones están integrados por 5 o menos autores. Nuevamente, se apunta al supuesto de que la colaboración tiene un efecto positivo sobre la producción científica, tal y como han revelado los resultados de los autores más productivos. No obstante, en el caso de los grupos más numerosos, se aprecia que los trabajos no se firman por todos o por la mayoría de los miembros del grupo. Es posible que la especialización de los miembros del grupo o que la organización interna de los mismos influya en el número de autores por trabajo.

\section{FIGURA 4}

Número de artículos según el tamaño del grupo

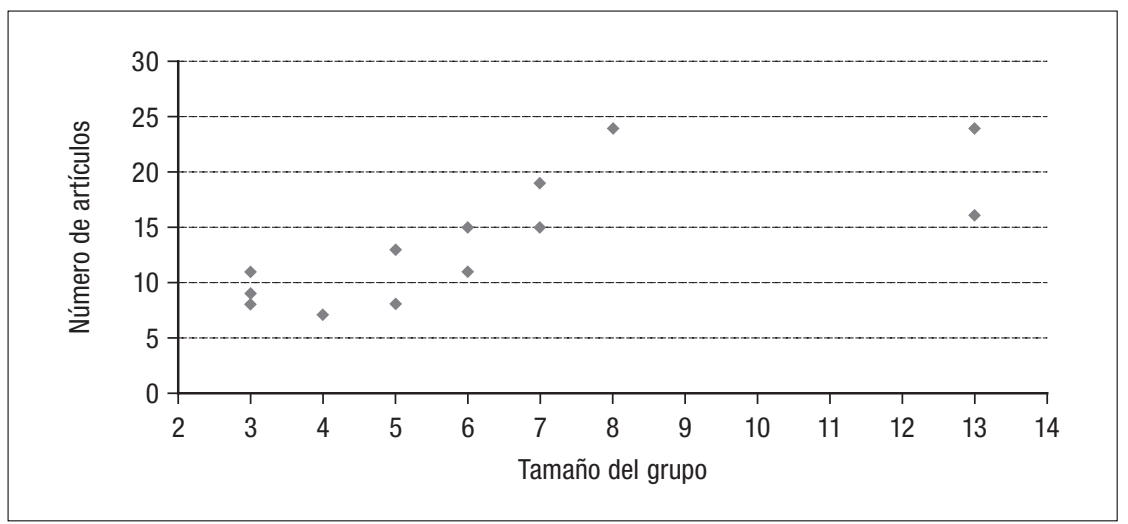

Adicionalmente, la cohesión de los grupos se analizó a través de la densidad. El número de miembros afecta a la cohesión del grupo, de manera que la colaboración entre los miembros se complica a medida que aumenta el tamaño de los grupos. En este estudio, la densidad (cuyo rango oscila entre 0 y 1 ) es máxima en aquellos grupos constituidos por cinco o menos investigadores, mientras que es menor en aquellos formados por seis o más miembros. Valderrama-Zurián (2007) obtuvo resultados similares al estudiar la colaboración entre investigadores españoles del ámbito de la Medicina, entre los cuales la densidad sólo alcanzó el máximo valor en los grupos de menos de 6 integrantes.

Los resultados indican que los grupos identificados desarrollan su actividad investigadora principal en torno a seis disciplinas de las CCAFD. La Fisiología y 
la Teoría del Entrenamiento son, cada una, el ámbito de trabajo de 8 grupos, la Medicina lo es de otros dos grupos, y la Biomecánica, la Sociología y la Psicología son disciplinas principales de sendos grupos de investigación.

\section{Conclusiones e implicaciones}

La producción científica en las CCAFD ha sido objeto de análisis durante los últimos años, pero aspectos como la colaboración científica apenas se han estudiado. En este trabajo, que ha analizado la colaboración a través de la coautoría reflejada en una muestra de revistas españolas del campo, se constata que la colaboración predomina sobre el trabajo individual de los investigadores tanto en el conjunto de la producción científica como en la mayoría de las revistas y de las disciplinas del las CCAFD. Ese predominio es muy evidente en el caso de los autores más productivos, donde se observan elevados niveles de colaboración. Estos resultados coinciden con la tendencia a la colaboración que se ha producido en las últimas décadas, que puede interpretarse como un síntoma de desarrollo científico. Ahora bien, la amplitud de esa colaboración, en cuanto al número de agentes implicados, es más bien reducida al producirse mayoritariamente entre dos o tres investigadores. Asimismo, los grupos identificados tienen un tamaño bastante reducido. Por tanto, sería recomendable que la comunidad científica española de las CCAFD extendiera su grado de colaboración e implicara a más investigadores en sus equipos. Ese crecimiento debería compatibilizarse con la cohesión suficiente de los equipos para evitar que las colaboraciones no derivaran en una simple yuxtaposición de investigadores o subgrupos.

En cualquier caso, es previsible que factores como la complejidad y amplitud de las investigaciones, el planteamiento de problemas que requieren un abordaje multidisciplinar, la conveniencia de compartir los costes de la investigación o la necesidad de constituir redes para alcanzar una proyección mayor, estimulen cada vez más la colaboración entre investigadores y grupos. Aunque la diversidad de culturas científicas que conviven en el campo de las CCAFD dificulta conocer cómo evolucionará la colaboración es previsible que, tanto por las razones anteriores como por los propios incentivos de la política científica, que premia la cooperación para la distribución y asignación de fondos, el número de agentes que investigan y acaban publicando juntos tienda a aumentar.

Las colaboraciones entre investigadores españoles y extranjeros, especialmente de países de habla inglesa, son muy limitadas, si bien es cierto que la muestra del estudio se reduce a revistas españolas y parte de las colaboraciones con investigadores de otros países pueden estar publicándose en títulos extranjeros de mayor visibilidad. En cualquier caso, el establecimiento de redes internacionales de colaboración debería constituir una prioridad en un campo de conocimiento emergente como éste. Esto exigiría incrementar sustancialmente las relaciones con investigadores de otros países y conferirles cierta estabilidad a través de proyectos u otras estrategias. En este sentido, tiene interés reforzar las colaboraciones 
con los países hispanohablantes y publicar en este idioma para contribuir al uso del español como lengua científica. Pero al mismo tiempo debe hacerse un esfuerzo por acrecentar las relaciones con investigadores de habla inglesa, entre otras cosas, para mejorar la proyección y la visibilidad de la comunidad científica española de las CCAFD. Paralelamente, las revistas españolas tienen que convertirse en instrumentos reconocidos suficientemente por los investigadores de otros países. Para ese propósito es imprescindible mejorar la visibilidad de las revistas en Internet y aprovechar las alternativas que este medio ofrece para la difusión de las contribuciones científicas, como el Directory Open Access Journals (DOAJ). Asimismo, deberían adoptarse estrategias como contar con la presencia de autores de prestigio internacional entre sus cuadros editoriales y establecer un uso indistinto del español y del inglés como lengua de publicación, además de acceder a programas institucionales de ayudas y financiación.

\section{Agradecimientos}

Agradecemos al Ministerio de Educación y Ciencia la ayuda recibida por el proyecto de investigación que sustenta el trabajo de este artículo (Proyecto I+D+I 2004-2007, SEJ2004-03996/EDUC).

\section{Referencias}

Aleixandre, R. y Galdón, R. (2003): Autoría y colaboración en los artículos publicados en revistas dermatológicas españolas (1996-2000). Piel, vol. 18 (6), 291-297.

Alonso-Arroyo, A.; Pulgarín, A., y Gil-Leiva, I. (2005): Estudio cienciométrico de la colaboración científica en la Universidad Politécnica de Valencia, España. Information Research, vol. 11 (1) paper 245. http://InformationR.net/ir/11-1/paper245.html [consulta: 20/02/2009].

Arunachalam, S., y Jinandra, M. (2000): Science in a small country at a time of globalization: domestic and international collaboration in new biology research in Israel. Journal of Information Science, vol. 26 (1), 39-49.

Bordons, M., y Gómez, I. (1997): La actividad científica española a través de indicadores bibliométricos en el período 1990-93. Revista General de Información y Documentación, vol. 7 (2), 69-86.

Bordons, M., y Gomez, I. (2000): Collaboration networks in science. In Cronin, B. y Atkins, H.B. (eds.), The web of knowledge: A Festschrift in honor of Eugene Garfield (pp. 197-213). Medford, NJ: Information Today, Inc. \& ASIS.

Bordons, M.; Zulueta, M. A.; Cabrero, A., y Barrigón, S. (1995): Identifying research teams with bibliometric tools. In M. E. Koening y A. Brookstein, (Eds), Proceedings of the Fifth Biennial Conference of the International Society for Scientometrics and Informetrics (pp. 83-92). Medford: Learned Information.

Borgman, C. L., y Furner, J. (2002): Scholarly Communication and Bibliometrics. Annual Review of Information Science and Technology, vol. 36, 3-72. 
Calatayud, F., y Balaguer, I. (2000): Estudio de los colegios invisibles en la revista Apunts (1964-1993). Apunts. Educación Física y Deportes, 61, 6-23.

Cronin, B. (2001): Hyperauthorship: A postmodern perversion or evidence of a structural shift in scholarly communication practices? Journal of the American Society for Information Science and Technology, vol. 52 (7), 558-569.

Cronin, B.; Shaw, D., y La Barre, K. (2003): A cast of thousands: coauthorship and subauthorship collaboration in the 20th century as manifested in the scholarly journal literature of psychology and philosophy. Journal of the American Society for Information Science and Technology, vol. 54 (9), 855-871.

Cronin, B.; Shaw, D., y La Barre, K. (2004): Visible, less visible, and invisible work: patterns of collaboration in 20th century Chemistry. Journal of the American Society for Information Science and Technology, vol. 55 (2), 160-168.

Drenth, J. P. H. (1998): Multiple authorship: The contribution of senior authors. Journal of the American Medical Association, vol. 280 (2), 219-221.

Glänzel, W. (2002): Coauthorship patterns and trends in the sciences (1980-1998): A bibliometric study with implications for database indexing and search strategies. Library Trends, vol. 50 (3), 461-473.

Glänzel, W.; Schubert, A., y Czerwon, H. J. (1999): A bibliometric analysis of international scientific cooperation of the European Union (1985-1995). Scientometrics, vol. 45 (2), 185-202.

Heinemann, K. (1990): 25 years of the International Review for the Sociology of Sport A content analysis. International Review for the Sociology of Sport, vol. 25 (1), 3-16.

Jiménez, E., y Moya, F. (1997): Análisis de la autoría en revistas españolas de Biblioteconomía y Documentación, 1975-1995. Revista Española de Documentación Científica, vol. 20 (3), 252-266.

Lidor, R.; Miller, U., y Rotstein, A. (1999): Is research on aging and physical activity really increasing? A bibliometric analysis. Journal of Aging and Physical Activity, vol. 7 (2), 182-195.

López, P., y Escalada, C. (1999): Veinte años de Sociología española a través de la "Revista Española de Investigaciones Sociológicas" (1978-1997). Revista General de Información y Documentación, vol. 9 (1), 161-173.

López, P.; Díaz, R. A.; Angulo, S., y Giménez, E. (2001): Estudio bibliométrico y de calidad de la Revista General de Información y Documentación. Revista General de Información y Documentación, vol. 11 (1), 11-32.

Newman, M. E. (2004): Coauthorship networks and patterns of scientific collaboration. Proceedings of the National Academy of Sciences of the United States of America, vol. 101(S1), 5200-5205.

Olmeda-Gómez, C.; Perianes-Rodríguez, A.; Ovalle-Perandones, M. A., y Moya-Anegón, F. (2008): Comparative analysis of university-government-enterprise co-authorship networks in three scientific domains in the region of Madrid. Information Research, vol. 13 (3) paper 352. http://InformationR.net/ir/13-3/paper352.html [consulta: 15/04/2009].

Otte, E., y Rousseau, R. (2002): Social networks analysis: a powerful strategy, also for the information sciences. Journal of Information Science, vol. 28 (6), 441-453.

Thompson, B. (1996): A review of the British Journal of Sports Medicine 1991-5. British Journal of Sports Medicine, vol. 30 (4), 354-355. 
Valderrama-Zurián, J. C.; González-Alcaide, G.; Valderrama-Zurián, F. J.; Aleixandre-Benavent, R., y Miguel-Dasit, A. (2007): Redes de coautorías y colaboración institucional en REVISTA ESPAÑOLA DE CARDIOLOGÍA. Revista Española de Cardiología, vol. 60 (2), 117-130.

Valenciano, J.; Devís-Devís, J., y Villamón, M. (2008a): Análisis comparativo de la calidad de las revistas científico-técnicas españolas de Ciencias de la Actividad Física y el Deporte (2000-2005). Information Research, vol. 13 (1), paper 337. http://informationr. net/ir/13-1/paper337.html [consulta: 15/05/2009].

Valenciano, J.; Devís-Devís, J., y Villamón, M. (2009): Autoría institucional de los trabajos publicados en revistas españolas de ciencias del deporte. Cultura, Ciencia y Deporte, vol. 5 (10), 7-14.

Valenciano, J.; Villamón, M., y Devís-Devís, J. (2008b): Evaluación y clasificación de las revistas científico-técnicas españolas de Ciencias de la Actividad Física y el Deporte. Revista Española de Documentación Científica, vol. 31 (3), 396-412.

Vallejo, M.; Ocaña, A.; Bueno, A.; Torralbo, M., y Fernández, A. (2005): Producción científica sobre Educación Multicultural contenida en las bases de datos Social Sciences Citation Index y Arts \& Humanities Citation Index (1956-2003). Revista Española de Documentación Científica, vol. 28 (2), 206-220.

Villamón, M.; Devís, J.; Valencia, S., y Valenciano, J. (2007): Características y difusión de las revistas científico-técnicas españolas de ciencias de la actividad física y el deporte. El Profesional de la Información, vol. 16 (6), 605-615.

Villamón, M.; Valenciano, J.; Devís-Devís, J., y Pérez-Samaniego, V. (2009): Las revistas españolas de ciencias del deporte incluidas en el "Catálogo Latindex". Movimento, vol. 15 (3), 13-34.

Wang, Y.; Wu, Y. S.; Pan, Y. T.; Ma, Z., y Rousseau, R. (2005): Scientific collaboration in China as reflected in co-authorship. Scientometrics, vol. 62 (2), 183-198.

Ward, P., y Ko, B. (2006): Publication trends in the Journal of Teaching in Physical Education from 1981 to 2005. Journal of Teaching in Physical Education, vol. 25 (3), 266-280.

Yaman, H., y Atay. E. (2007): PhD theses in Turkish sports sciences: A study covering the years 1988-2002. Scientometrics, vol. 71 (3), 415-421.

Zulueta, M. A.; Cabrero, A., y Bordons, M. (1999): Identificación y estudio de grupos de investigación a través de indicadores bibliométricos. Revista Española de Documentación Científica, vol. 22 (3), 333-347. 